\title{
An ESPResSo implementation of elastic objects immersed in a fluid
}

\author{
I. Cimrák ${ }^{1 a}$, M. Gusenbauer ${ }^{2 b}$, I. Jančigová ${ }^{3 c}$ \\ ${ }^{a}$ Department of Software Technologies, Faculty of Management Science and Informatics, University of Žilina, \\ Slovakia \\ ${ }^{b}$ St. Poelten University of Applied Sciences, Industrial Simulation, St. Poelten, Austria \\ ${ }^{c}$ Department of InfoCom Networks, Faculty of Management Science and Informatics, University of Žilina, Slovakia
}

\begin{abstract}
We review the lattice-Boltzmann (LB) method coupled with the immersed boundary (IB) method for the description of combined flow of particulate suspensions with immersed elastic objects. We describe the implementation of the combined LB-IB method into the open-source package ESPResSo. We present easy-to-use structures used to model a closed object in a simulation package, the definition of its elastic properties, and the interaction between the fluid and the immersed object. We also present the test cases with short examples of the code explaining the functionality of the new package.
\end{abstract}

Keywords: ESPResSo, molecular simulation, blood flow modeling, lattice-Boltzmann method, immersed boundary method

2010 MSC: 92C37, 68U20

\section{FRAMEWORK FEATURES}

\section{Feature}

Templates

geometry

elastic properties

\section{Objects}

source template

position, rotation

Properties

position

external forces

deformation

\section{OIF command example and options}

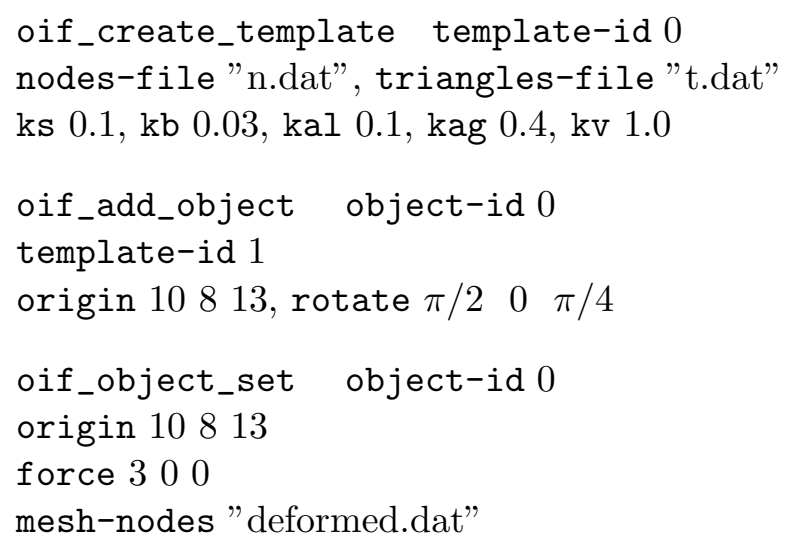

\footnotetext{
${ }^{1}$ The work of I. Cimrák was supported by the Slovak Research and Development Agency under the contract No. APVV-0441-11, and by the Marie-Curie grant No. PCIG10-GA-2011-303580.

${ }^{2}$ M. Gusenbauer was supported by the NÖ Forschungs- und Bildungsges.m.b.H. (NFB) through the Life Science Calls.

${ }^{3}$ The work of I. Jančigová was supported by the Slovak Research and Development Agency under the contract No. APVV-0441-11.
}

Preprint submitted to Computer Physics Communications

December 13, 2013 


\begin{tabular}{ll}
$\begin{array}{l}\text { Output } \\
\text { vtk visualization } \\
\text { deformation }\end{array}$ & $\begin{array}{l}\text { oif_object_output object-id } 0 \\
\text { vtk-pos "pos.vtk" } \\
\text { mesh-nodes "deformed.dat" }\end{array}$ \\
$\begin{array}{l}\text { Mesh } \\
\text { mesh files }\end{array}$ & $\begin{array}{l}\text { oif_check_mesh } \\
\text { nodes-file "n.dat", triangles-file "t.dat" } \\
\text { mesh analysis } \\
\text { mesh repair }\end{array}$ \\
$\begin{array}{l}\text { orientation } \\
\text { repair "r.dat" }\end{array}$ \\
$\begin{array}{l}\text { initialization } \\
\text { information }\end{array}$ & oif_init \\
\hline
\end{tabular}

\section{Introduction}

The simulations of flow with immersed objects are a key technique for analysis in numerous areas. One can simulate the propulsion of bacteria [1], another application is the analysis of fish-like behavior [2]. Our current research is motivated by biomedical applications for which simulation of blood on the level of blood cells immersed in a fluidic blood plasma is crucial (Fig. 1). These applications require understanding of processes depending on individual behavior of particular cells. Among others, we speak about microfluidic devices aimed for filtering of circulating tumor cells from blood [3].

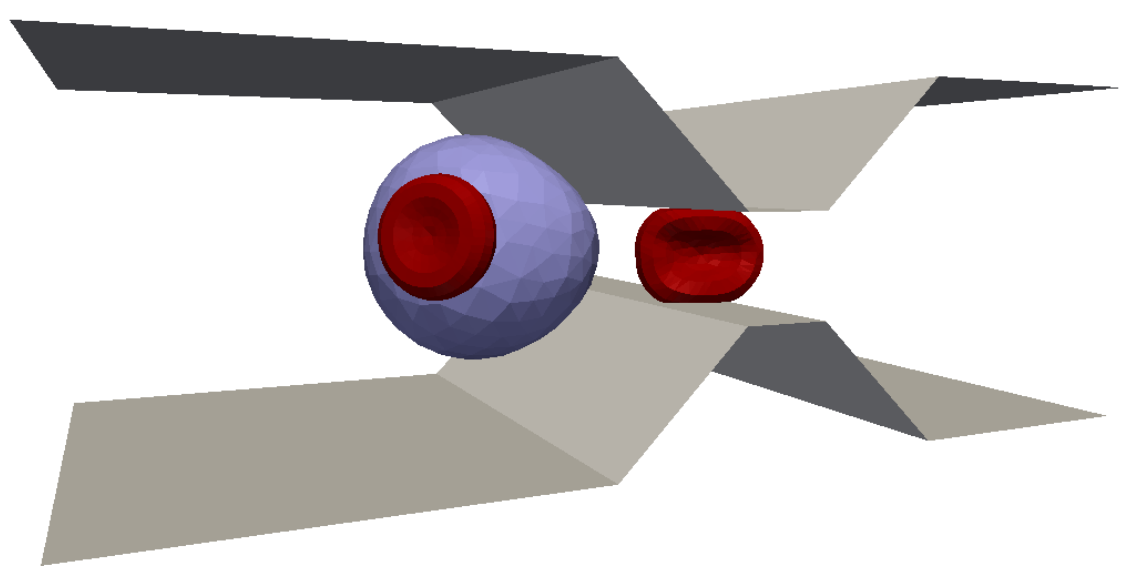

(a)

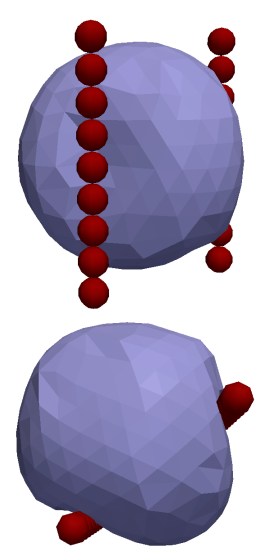

(b)

Figure 1: Elastic objects in biomedical applications a) Simulation of blood flow through tunable magneto-active polymer channel [4] b) Circulating tumor cell captured in magnetic bead trap (side and top view) [5]

We utilize the lattice-Boltzmann (LB) method for the description of the fluid dynamics and the immersed boundary (IB) method for the description of the immersed objects. The coupling of both methods provides an accurate description of the complete fluid-structure interactions.

The lattice-Boltzmann method is a powerful technique enabling fast and accurate computation of the fluid flow in complex structures $[6,7,8]$. In its nature, it is suitable for parallelization, 
performing very well in the benchmark examples $[9,10,11]$. The LB method describes the flow dynamics by giving information about the fluid velocity and pressure in each space-time discretization point of the computational domain.

The immersed boundary (IB) method is based on the discretization of the boundary of the immersed object. The method was first introduced by Charles S. Peskin in 1972 [12]. The advantage of the method lies in its ability to capture the changes in the shape of the object without re-meshing and adaptive refinement of the underlying meshes. This is a crucial advantage for the simulation of moving boundaries.

\section{Method}

The ESPResSo package is aimed for soft-matter simulations. Originally, it was intended for simulations of different chemical systems where molecules and atoms are involved. These atoms are modeled as particles with their own mass. User defines bonds and potentials that generate forces between the particles. Consequently, particles move in the space subject to these forces and numerous other constraints.

In later releases of ESPResSo, the LB method was implemented to simulate soft-matter systems immersed in fluid. However, the package had no concept of a closed object. All the entities simulated in ESPResSo were open, tree-like structures without inner volume and surface. Our idea was to extend the functionality of ESPResSo by introducing closed objects with their own particle management in order to simulate elastic objects immersed in a fluid.

In this section, we first briefly describe the LB method as it was implemented in the original version of ESPResSo. Then we describe the IB method that we have implemented.

Lattice Boltzmann method: This method is based on fictive particles. These particles perform consecutive propagations and collisions over a fixed discrete lattice. Consider a lattice placed over the three-dimensional domain and consisting of identical cubic cells. This lattice creates an Eulerian grid which is fixed during the entire simulation. The variable of interest in the LB method is $n_{i}(x, t)$ which is the particle density function for the lattice point $x$, discrete velocity vector $e_{i}$, and time $t$. We use the D3Q19 version of the LB method (three dimensions with 19 discrete directions $e_{i}$, so $\left.i=0, \ldots, 18\right)$. The governing equations in the presence of external forces, are

$$
\underbrace{n_{i}\left(x+e_{i} \delta_{t}, t+\delta_{t}\right)=n_{i}(x, t)}_{\text {propagation }}-\underbrace{\Delta_{i}(\mathbf{n}(x, t))}_{\text {collision }}+\underbrace{f_{i}(x, t)}_{\text {externalforces }}
$$

where $\delta_{t}$ is the time step and $\Delta_{i}$ denotes the collision operator that accounts for the difference between pre- and post-collision states and satisfies the constraints of mass and momentum conservation. $f_{i}$ is the external force exerted on the fluid. We refer to [13] and [14] for details on the LBM. The macroscopic quantities such as velocity $u$ and density $\rho$ are evaluated from

$$
\rho(x, t)=\sum_{i} n_{i}(x, t) \quad \text { and } \quad \rho(x, t) u=\sum_{i} n_{i}(x, t) e_{i} .
$$

Immersed Boundary method: This method is based on the discretization of object's boundary. The boundary is covered by a set of IB points linked by triangular mesh, which is called Lagrangian mesh. The positions of IB points are not restricted to any lattice. To take the mechanoelastic properties of the immersed objects into account, geometrical entities in this mesh (edges, 
faces, angles between two faces, ...) are used to model stretching, bending, stiffness, and other properties of the boundary. They define forces according to the current shape of the immersed object that are exerted on IB points. These forces cause motion of the IB points according to the Newton's equation of motion

$$
m \frac{d^{2} X_{j}}{d t^{2}}=F_{j}
$$

where $m$ is the mass of the IB point, $X_{j}$ is the position and $F_{j}$ is the force exerted on the particular IB point $j$. The source of $F_{j}$ is either from the above mentioned elasto-mechanical properties of the immersed object, or from the fluid-structure interaction.

Equations (1) and (2) describe two different model components on two different meshes: the motion of the fluid and the motion of the immersed objects. For coupling, ESPResSo uses an approach from [13] with a drag force that is exerted on a sphere moving in the fluid. Analogous to the Stokes formula for the sphere in a viscous fluid, we assume the force exerted by the fluid on one IB point to be proportional to the difference of the velocity $v$ of the IB point and the fluid velocity $u$ at the same position,

$$
F_{j}=\xi(v-u) .
$$

Here $\xi$ is a proportionality coefficient which we will refer to as the friction coefficient. In the previous expression, the velocities $v$ and $u$ are computed at the same spatial location, whereas we posses $u$ in fixed Eulerian grid points and $v$ in moving Lagrangian IB points. Therefore, for computation of $u$ at the IB point, we use linear interpolation of the $u$ values from nearby fixed grid points.

There is also an opposite effect: not only fluid acts on the IB point, but also the IB point acts on the fluid. Therefore, the opposite force $-F_{j}$ needs to be transferred back to the fluid. $F_{j}$ from the location of the IB point is distributed to the nearest grid points. Distribution is inversely proportional to the cuboidal volumes with opposite corners being the IB point and the grid point [13].

\section{Object-in-fluid framework}

We implement an object-in-fluid (OIF) framework into ESPResSo that allows the use of objects with inner volume, for example blood cells, magnetic beads, capsules and so on. We use LB-IB method where IB points from the triangulation are identified with ESPResSo particles. The edges of the triangular mesh are identified as bonds in ESPResSo. Bonds generate elastic forces that keep the shape of the object. The movement of the object is achieved by applying calculated forces to the IB points.

The immersed objects are composed of a membrane encapsulating the fluid inside the object. For now, the inside fluid must have the same density and viscosity as the outside fluid.

The original ESPResSo works with particles that can be defined by a single part command executed for each particle. Particles are virtually connected with bonds executing inter and part bond commands. To create a mesh, e.g. a triangular mesh with 300 mesh nodes covering a sphere, one needs to execute a series of commands analyzing the mesh, computing hundreds of edge lengths, all angles between triangles, etc. Afterwards, more than thousand commands must be executed to add the particles and define the bonds. In the OIF framework we enable the automatization of this process with numerous features. 
The OIF framework works in two steps. First, one needs to create a template, then one can add actual objects. The template has its own elastic properties and shape. Templates are not real objects, they only keep information about the objects to be created. After a template (or several templates) is created, one can add actual objects to the simulation by specifying the template from which the object takes its geometry and elastic properties, the location and, optionally, the rotation of the object.

During the simulation, one can analyze the objects by printing their physical properties. Also, different quantities characterizing the objects can be set.

Further functionality includes analysis of the mesh files, output for the VTK visualization using a standalone visualization software, e.g. ParaView [15]. Detailed description of all currently implemented features of the OIF framework is in Section 5. However, the OIF framework is a lively project that is being extended on regular base. Therefore for up-to-date documentation on the latest developments we recommend the ESPResSo documentation [16].

\section{Elastic interactions}

The generic implementation of bonded interactions in ESPResSo is described in detail in [17, 18]. Bonded interactions are always defined between two, three or sometimes four points. They generate forces that are exerted on each IB point. These forces must be evaluated at a certain point of the integration algorithm. At that moment, with given positions of the IB points, the forces for each specific interaction can be calculated and added to the IB points.

Parameters: There are several parameters involved in this model. All of them should be calibrated with respect to the application.

Mass of the particles: Every particle has its mass that influences the dynamics. Its default value is one, however it can be changed when adding the object into simulation with oif_add_object mass command.

Friction coefficient $\xi$ : The parameter describing the strength of fluid-particle interaction is the friction parameter from the original ESPResSo command lbfluid, see (3).

Parameters of elastic moduli: Elastic behavior can be described by five different elastic moduli: stretching, bending, local and global area preservation and volume preservation. Each of them has its own scaling parameter: $\mathrm{ks}, \mathrm{kb}, \mathrm{kal}, \mathrm{kag}, \mathrm{kv}$.

The mass of the particles and the friction coefficient can be calibrated using the known analytical values of the drag coefficients for ellipsoidal objects. More details about the calibration is given in [19].

The elastic parameters depend on the way how the physical properties are modeled. For each physical property there are always numerous approaches how to model that particular property. As a very simple example let us discuss the local area conservation. Assume an elastic object has a relaxed shape, in which this objects remains unless external forces are applied. In the relaxed shape, each local part of the surface has its area. The general idea is that once the local part of the object's surface has a larger area than in the relaxed state then this part must be shrunk. If the current area is smaller than that in the relaxed shape, the surface must be expanded.

To implement this general idea we show two different approaches. Both approaches first compute the magnitude of the force that will be applied to each vertex of the triangles in the mesh. 
This magnitude is proportional to the difference between the current area of the triangle and the area in the relaxed shape. The directions of the forces however differ in the two approaches. First approach puts the forces in the direction from the vertex to the centroid of the triangle. The second approach applies the forces in the vertices in the direction of the respective altitudes of the triangle. Both approaches follow the main idea, however the actual effect on the triangle is different.

In the current version of the OIF framework, we work with the elastic models taken from [20]. More details about the mechanical and biological aspects specifically for red blood cells are presented in the cited work. The proper calibration to fit the experimental data has been performed in $[19,21]$.

However, we are aware of the different approaches. Authors in $[22,23,24]$ use the energy approach where the forces are derived from the energy contributions rather than defined explicitly as in [20]. The OIF framework is flexible enough to implement any method based on the application of forces to the nodes in the triangular mesh.

The following interactions are implemented in order to mimic the mechanics of elastic or rigid objects immersed in the LB fluid flow. They are all based on the fact that an elastic object without any external forces has a relaxed shape. The object always tends to recover this relaxed shape. Once this shape is deformed, elastic forces act on the boundary of the object, trying to return it to its relaxed state. So for example, all edges of the triangulation in the relaxed shape have a reference length which we call relaxed length. Similarly, there is a relaxed angle between two incident triangles in the mesh, a relaxed area of each triangle in the mesh and so on.

The elastic forces have specific expressions for each elastic modulus. Their mathematical formulations have been taken from [25].

a)
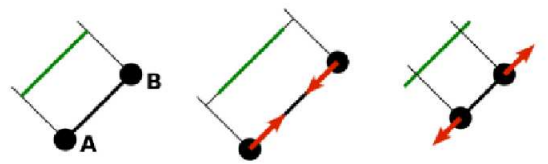

b)

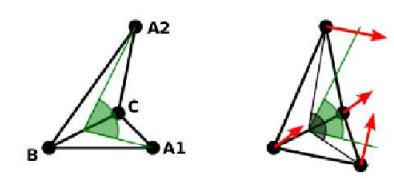

c)
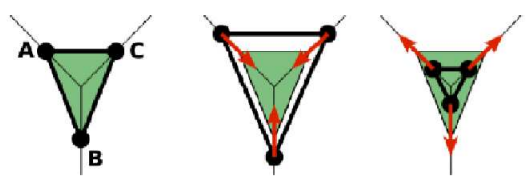

d)
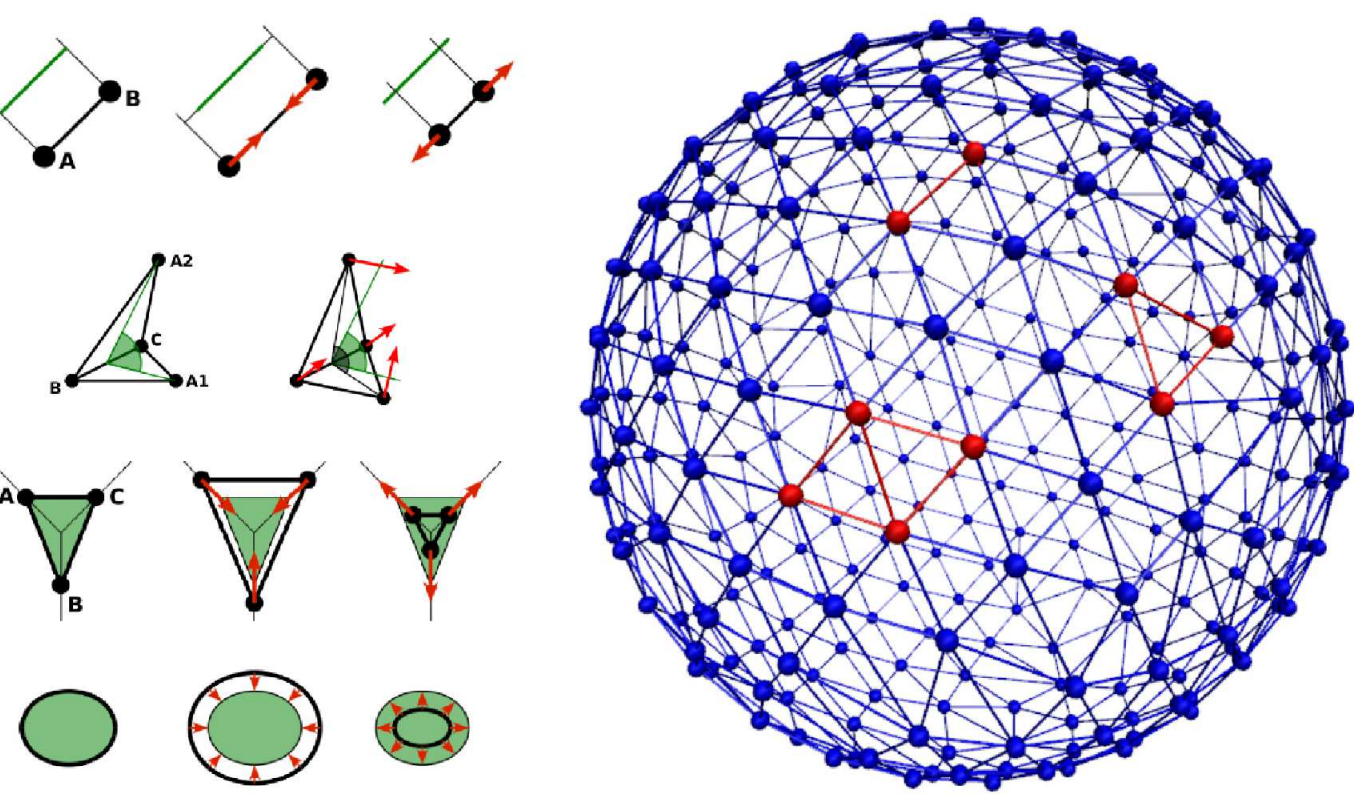

Figure 2: Elastic sphere model in ESPResSo:

a) stretching force, b) bending force, c) local area force, d) volume force/global area force 


\section{Stretching force}

This type of interaction is available for closed 3D immersed objects as well as for $2 \mathrm{D}$ sheet flowing in the $3 \mathrm{D}$ flow.

It forces each triangulation edge to adjust towards its relaxed length. For each edge of the mesh, $L$ is the current length of the edge. By $L^{0}$ we denote the relaxed length. In the case of $L=L^{0}$ no forces are added. $\Delta L=L-L^{0}$ denotes the deviation from the relaxed length. The stretching force acting on edge endpoints is computed using

$$
F_{\mathrm{s}}=k_{\mathrm{s}} \kappa(\lambda) \frac{\Delta L}{L^{0}} n .
$$

Here, $n$ is the unit vector pointing from the one edge endpoint to the other, $k_{\mathrm{s}}$ is the stretching constant, $\lambda=L / L^{0}$, and $\kappa$ is a nonlinear function that resembles neo-Hookean behavior

$$
\kappa(\lambda)=\frac{\lambda^{0.5}+\lambda^{-2.5}}{\lambda+\lambda^{-3}} .
$$

For linear behavior of the stretching force we simply have $\kappa=1$. The stretching force acts between two particles and is symmetric (Figure 2a).

\section{Bending force}

Beside the stretching force, the tendency of an elastic object to maintain the relaxed shape is governed also by prescribing the preferred angles between two incident triangles of the mesh. This type of interaction is available for closed 3D immersed objects, as well as for $2 \mathrm{D}$ sheet flowing in the $3 \mathrm{D}$ flow.

The angle between two triangles in the resting shape is denoted by $\theta^{0}$. For closed immersed objects, one always has to set the inner angle. The deviation of this angle $\Delta \theta=\theta-\theta^{0}$ is computed and defines the bending force for each triangle.

$$
F_{\mathrm{b}}=k_{\mathrm{b}} \frac{\Delta \theta}{\theta^{0}} n
$$

Here, $n$ is the unit normal vector to the triangle and $k_{\mathrm{b}}$ is the bending constant. The force $F_{\mathrm{b}}$ is assigned to the two vertices not belonging to the common edge. The opposite force divided by two is assigned to the two vertices lying on the common edge (Figure $2 \mathrm{~b}$ ).

Unlike the stretching force, the bending force is strictly asymmetric. For more details, see the ESPResSo documentation [16]. Notice, that concave objects can be defined as well. If $\theta_{0}$ is larger than $\pi$, then the inner angle is concave.

\section{Local area conservation}

This interaction conserves the area of the triangles in the triangulation. This type of interaction is available for closed 3D immersed objects, as well as for 2D sheet flowing in the 3D flow.

The deviation of the triangle surface $S$ is computed from the triangle surface in the resting shape $\Delta S=S-S^{0}$. The area constraint assigns the following shrinking/expanding force to every vertex

$$
F_{\mathrm{al}}=-k_{\mathrm{al}} \frac{\Delta S}{S} w
$$


where $k_{\text {al }}$ is the area constraint coefficient, and $w$ is the unit vector pointing from the centroid of triangle to the vertex. Similarly, analogical forces are assigned to other vertices of the triangle (Figure 2c). This interaction is symmetric.

\section{Global area conservation}

This type of interaction is available for closed 3D immersed objects, as well as for 2D sheet flowing in the $3 \mathrm{D}$ flow.

The conservation of local area is sometimes too restrictive. The current surface of the whole immersed object is denoted by $S_{g}$, the surface in the relaxed shape by $S_{g}^{0}$ and $\Delta S_{g}=S_{g}-S_{g}^{0}$. The global area conservation force is then defined as

$$
F_{\text {ag }}=-k_{\text {ag }} \frac{\Delta S_{g}}{S_{g}} w
$$

The force $F_{\text {ag }}$ is assigned to all vertices of the object (Figure 2d).

Unlike the previous three forces (and related bonds), this one is different from classical bonds in ESPResSo, because it requires an advance calculation on the scale of the whole immersed object - to get its total area. This calculation has been implemented to allow for proper area computation also in parallelized simulations when parts of the given object lie in different blocks of the decomposed domain. In particular, consider a case when the immersed object lies in several regions that belong to different computational nodes. To compute the surface of such immersed object, each computational node needs to compute partial surface of the corresponding part and send the information to master to gather the whole surface of the object. To implement this, at each surface computation, a message is broadcasted to all computational nodes that adds the partial surface of the object. After gathering the information from all nodes, we can compute the actual forces for the global area conservation.

\section{Volume conservation}

This type of interaction is available solely for closed 3D immersed objects.

The deviation of the object's volume $V$ is computed from the volume in the resting shape $\Delta V=V-V^{0}$. For each triangle the following force is computed

$$
F_{\mathrm{v}}=-k_{\mathrm{v}} \frac{\Delta V}{V^{0}} S n
$$

where $S$ is the area of triangle, $n$ is the normal unit vector of triangle's plane, and $k_{\mathrm{v}}$ is the volume constraint coefficient. The volume of the immersed object is computed from

$$
V=\sum_{\text {triangles }} S n \cdot h
$$

where the sum is computed over all triangles of the mesh and $h$ is the unit vector from the centroid of triangle to a plane which does not cross the object. The force $F_{\mathrm{v}}$ is equally distributed to all three vertices (Figure 2d).

Similar to the computation of global area force, the volume force also requires a prior calculation on the scale of the whole object - to get the total volume. And again, it is implemented in such a way that partial volumes are added together in parallelized simulations when the object belongs to more than one block of decomposed domain. 


\section{Remark}

The actual values of $k_{\mathrm{s}}, k_{\mathrm{b}}, k_{\mathrm{al}}, k_{\mathrm{ag}}, k_{\mathrm{v}}$ need to be calibrated for each type of the elastic object separately. They strongly depend on the physical values of elastic moduli. Their values can be determined e.g. from stretching experiments performed with red blood cells [20]. The stretching force expression (4) yields the sensitivity of $k_{\mathrm{S}}$ on the density of the triangular mesh. This dependence was elaborated in [21].

\section{Implementation details and OIF command description}

All commands and variables in the OIF framework start with prefix oif_. Syntax in the OIF framework follows the structure from ESPResSo. Each OIF command consists of command keyword followed by several option - arguments couples. The order of these couples is arbitrary. Some options of the commands are mandatory, some are optional. The options mostly have one or more arguments but there are also options without arguments.

The mandatory options are listed directly after the command keyword, the optional ones are listed inside brackets.

\section{Initialization, general info}

$$
\begin{aligned}
& \text { oif_init } \\
& \text { oif_info }
\end{aligned}
$$

The first command initializes the OIF framework and must be used before any other OIF commands. It creates global variables used in the OIF framework. These variables are accessible on the Tcl level in ESPResSo, but for the ordinary user of the OIF framework it is not necessary to access them. The detailed description of the OIF variables is in the ESPResSo documentation.

The second command prints basic information about the current number of created templates and objects, etc.

Both commands are without options.

\section{Template creation}

oif_create_template template-id tid nodes-file nfile

triangles-file tfile [stretch sx sy sz]

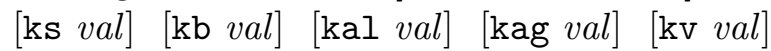

template-id Each template must have its unique identifier tid. This identifier starts from 0 and must be numbered consecutively.

nodes-file, triangles-file The information about the geometry is extracted from a surface triangulation called mesh. Spatial positions of the mesh points are obtained from the ifile. This simple text file contains lines and each line has three spatial coordinates of a mesh point. These coordinates should be centered in such a way that the approximate center of the object is located at the origin of the coordinate system. The order of mesh points in nfile defines the IDs of the points starting from 0 . Further, a file tfile contains lines and each line has three integer numbers representing the IDs of three mesh points in one triangular face of the mesh. It is crucial that three IDs in one line of tfile are in the right order. They must fulfill the rule that the normal vector of the mesh triangle must point inside the object. Specifically, 
assume A, B and $\mathrm{C}$ are three points of a triangle in the mesh and their IDs are 3,5 and 8. Compute the cross product $n=A B \times A C$. If the resulting vector $n$ (which is perpendicular to the triangle $A B C$ ) points inside the object then we have line 358 in tfile. If the vector $n$ points outside the object, we change the order of $\mathrm{B}$ and $\mathrm{C}$ and we have line 385 in tfile.

stretch The geometry described in nfile and tfile can be customized by stretching of the coordinates. This option will stretch $\mathrm{x}$-coordinates by factor $s x, \mathrm{y}$-coordinates by factor $s y$, and z-coordinates by factor $s z$.

$\mathrm{ks}, \mathrm{kb}, \mathrm{kal}, \mathrm{kag}, \mathrm{kv}$ These options define the prefactors for elastic forces.

- ks sets the stiffness of the spring between two particles on each edge of triangulation mesh. This parameter controls the stretching of the object.

- $\mathrm{kb}$ sets the stiffness of the spring between two triangles with common edge. This parameter controls the bending of the object.

- kal controls the conservation of the area of triangles.

- kag controls conservation of the total surface area of the immersed object.

- kv controls the conservation of the total volume of the immersed object.

More detailed explanation on different types of elastic forces is in Section 4.

\section{Adding of the object into simulation}

oif_add_object object-id oid template-id tid origin ox oy oz part-type ptype [rotate $r x$ ry $r z$ ] [mass val]

object-id Each object must have its unique identifier oid. This identifier starts from 0 and must be numbered consecutively.

template-id The geometry and elastic properties of each object are copied from a template identified by tid.

origin The object is placed in the space such that the approximate center of the object has coordinates $(o x, o y, o z)$.

part-type In ESPResSo, every particle has its type and this type is used for interactions, e.g. with the walls, etc. In the OIF framework, all particles that form one object have the same part-type ptype. Note, that two different objects may have the same part-type.

rotate Each object can be rotated. This does not influence the geometry and the elastic properties. The object will be rotated by $r x$ radians around the $\mathrm{x}$ axis. Similarly for $r y$ and rz.

mass Sets the mass of particles for this object.

\section{Visualization and data output}

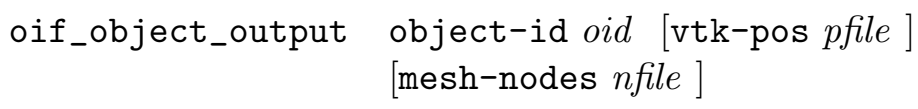


object-id Identifies the object under the consideration.

vtk-pos Outputs the triangular mesh of the object into the VTK-file $p$ file. This file is directly readable e.g. with ParaView [15] visualization software.

mesh-nodes Outputs the current shape of the object into the file nfile. This file can be used as an input for OIF command oif_object_set, option mesh-nodes. This option is used to start simulation from some deformed shape.

\section{Object analysis}

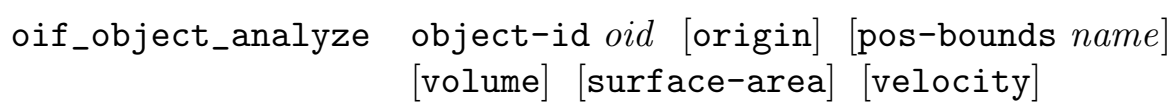

object-id Identifies the object under the consideration.

origin Prints the coordinates of the object's center. Since the object can be deformed, the coordinates of the origin are computed as an average of coordinates of all mesh points.

pos-bounds Computes six extremal coordinates of the object. More precisely, runs through all the mesh points and remembers the minimal and maximal $\mathrm{x}$-coordinate, $\mathrm{y}$-coordinate and z-coordinate. If b-name is one of these: "z-min, z-max, $x$-min, $x$-max, y-min, y-max", then the procedure returns the corresponding value. If $b$-name is "all", then the procedure returns a list of six numbers.

volume Returns the volume of the object.

surface-area Returns the surface area of the object.

velocity Returns the velocity of the object computed as an average over the velocities of all mesh points.

\section{Object customization}

oif_object_set object-id oid [force $f x f y f z$ ] [origin ox oy oz] [mesh-nodes nfile]

object-id Identifies the object under the consideration.

force Sets the force vector $(f x, f y, f z)$ to all mesh nodes of the object. Setting is done executing ESPResSo command part $i$ set ext_force $f x f y f z$ for each particle of the object.

origin The object is translated so that its center coincides with the spatial point (ox,oy,oz).

mesh-nodes Providing the file nfile one can change the current shape of the object.

\section{Mesh analysis}

Sometimes it happens that the orientations of the triangles in tfile are not correct. To check whether one has the correct orientations or to fix some wrong ones, the following OIF command can be used. 


\begin{tabular}{|llll|}
\hline n.dat: & & & t.dat: \\
1.00000 & 0.00000 & 0.00017 & 91711 \\
0.99262 & 0.00000 & 0.12129 & 181013 \\
0.99266 & 0.00000 & -0.12096 & 195 \\
0.95106 & 0.16995 & 0.00000 & 1027 \\
0.95106 & -0.16995 & 0.00000 & 013 \\
0.94356 & 0.16995 & 0.11920 & 203 \\
& & & 0
\end{tabular}

Table 1: Example of geometry files.

oif_mesh_analyze nodes-file nfile triangles-file tfile

[orientation] [repair rfile] [flip rfile]

nodes-file,triangles-file The files that need to be checked or repaired.

orientation Checks whether all triangles have correct orientation.

repair Checks each triangle and corrects its orientation when necessary. Outputs the corrected incidence file rfile. Note that this works for the convex objects and for some concave objects.

flip Flips the orientation of all triangles and outputs new incidence file rfile.

This description of the OIF framework is not intended to be complete. There are different demands on extension of the code and the current version included in the latest release of ESPResSo is always described in the user guide available at [16].

\section{Example: simulation blood cells flow}

The following example is part of our research focus in biomedical applications. Isolation of circulating tumor cells using antibody covered surfaces requires a high contact probability of blood cells and the activated surfaces (see $[4,5]$ for details). In microfluidics, the blood flow is usually laminar, therefore cells follow the streamlines, without even touching the surfaces. Obstacles in the channel help increase the contact with blood cells.

In Table 2, an example of a simple script is presented. The intention is to show that with a very simple script one can simulate quite complex physical system of the blood cells flowing inside a microfluidic channel.

For the geometry of a red blood cell we use a triangulation with 400 nodes and 796 triangles. Triangulation is given in files $\mathrm{n}$. dat and $\mathrm{t}$.dat. First couple of lines from each file are presented in Table 1.

Initialization, lines 1-4. In the first three lines of the script, several mandatory arguments for ESPResSo are set. In general, our calculations have been done with keeping the time_step (line 1) for IB calculations equal to the tau (line 22) for fluid propagation. Even though it is possible to thermalize particles in ESPResSo (using the thermostat command, line 3), 


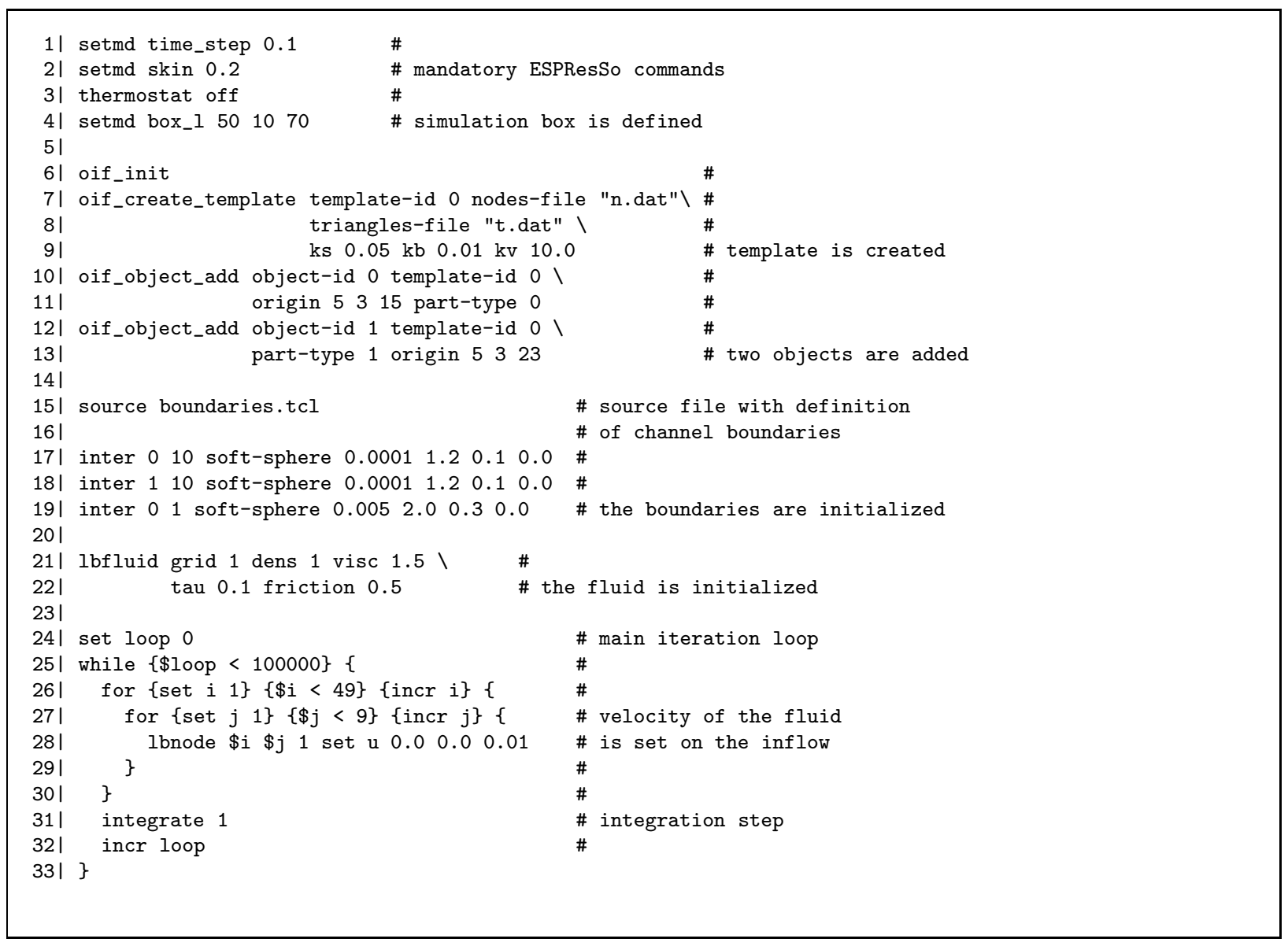

Table 2: Tcl script with simulation of flow of two red blood cells in a microfluidic channel. 
this is not relevant for simulations of objects on the scale of micrometers (e.g. blood cells), where the IB particles are not subject to thermal fluctuations. In line 4 the dimensions of the simulation box are given.

OIF framework. lines 6-13. Then we initialize the OIF framework with oif_init command. Further we create the template with template-id 0 by providing two mesh files and defining the elastic parameters. In this case we use the stretching, bending and volume conservation. The values for the elasticity parameters need to be determined from the modeled application.

Further, we add two objects, created from template 0 . We put them on different spatial locations specifying the coordinates of the origin. We set different part-type for both objects so that we can set the collision detection between them. Note, that the parameters can be given in any order.

Boundaries, lines 15-19. In line 15, we include an external Tcl script with definition of boundaries. We create a three-dimensional microfluidic channel with obstacles using the original ESPResSo commands constraint rhomboid, and lbboundary rhomboid, see Figure 3. The external script contains standard Tcl and ESPResSo commands.

Further, in lines 17-19 we set the repulsive interactions to simulate object-object and objectwall collisions.

Fluid init and main loop, lines 21-33. In lines 21-22 we initialize the fluid in the simulation. It is a standard ESPResSo command. Next we run the main loop. In each iteration, we first set the velocity of the fluid on the inflow to be constant (lines 26-30) using standard ESPResSo command lbnode and then we execute the integration procedure with ESPResSo command integrate.Currently, we are exploring the option to set the fluid velocity using "moving" boundaries, e.g. a lbboundary rhomboid that performs a modified bounce back boundary condition for the fluid. This way of prescribing the fluid velocity is faster because the actual computer operations are done on the $\mathrm{C}$ level and not on the Tcl level.

\section{Current computational capabilities}

For simulations of blood flow using the OIF framework, we need to make a compromise between the accuracy of blood cell model and the computational capabilities. It is not reasonable to have too detailed model of the red blood cell with thousands of boundary points. From our experience it is sufficient to use several hundreds of IB points to get the proper elastic behavior.

Our model is composed of two components, the fluid and the immersed objects. For both components a specific computational module is involved: For the fluid it is the lattice-Boltzman method with a fixed number of grid points, and for the immersed objects, it is a Newton equations integrator, with each immersed object discretized. In the LB method the simulation box is discretized by a regular lattice with $n_{x}, n_{y}, n_{z}$ being the size of the LB lattice in particular direction. That means that the number of unknowns in the LB method is proportional to $n_{x} \times n_{y} \times n_{z}$. The IB method is discretized by the triangular mesh so that the size of the problem depends on the size of the mesh $n_{i b}$ and on the number of immersed objects $n_{o b j}$. In total, the number of unknowns in the IB method is proportional to $n_{o b j} \times n_{i b}$.

The overall computational complexity thus scales with

$$
n_{x} \times n_{y} \times n_{z}+n_{o b j} \times n_{i b}
$$


From the previous expression one can make a clear picture of the interplay between the complexity levels on both components of the model. For our preliminary simulations we use a triangular mesh with 393 IB points. For illustration, on a standard notebook with a single core one can simulate the movement 5-10 blood cells in a channel of dimension 200x30x30 and the results are available in minutes. The length of the simulation is about $100 \mathrm{~ms}$.

On a stronger machine with 16 cores, $1.6 \mathrm{GHz}$ each, and a GPU, one is capable of simulating 100 blood cells during $100 \mathrm{~ms}$ time interval in a channel of dimension 300x200x50 and the results are available in hours. These figures are only for illustration.

Further improvements of the capabilities are readily possible by using stronger computational resources. The supercomputers containing several thousands of cores enable computation of hundreds or thousands of red blood cells in larger domains.

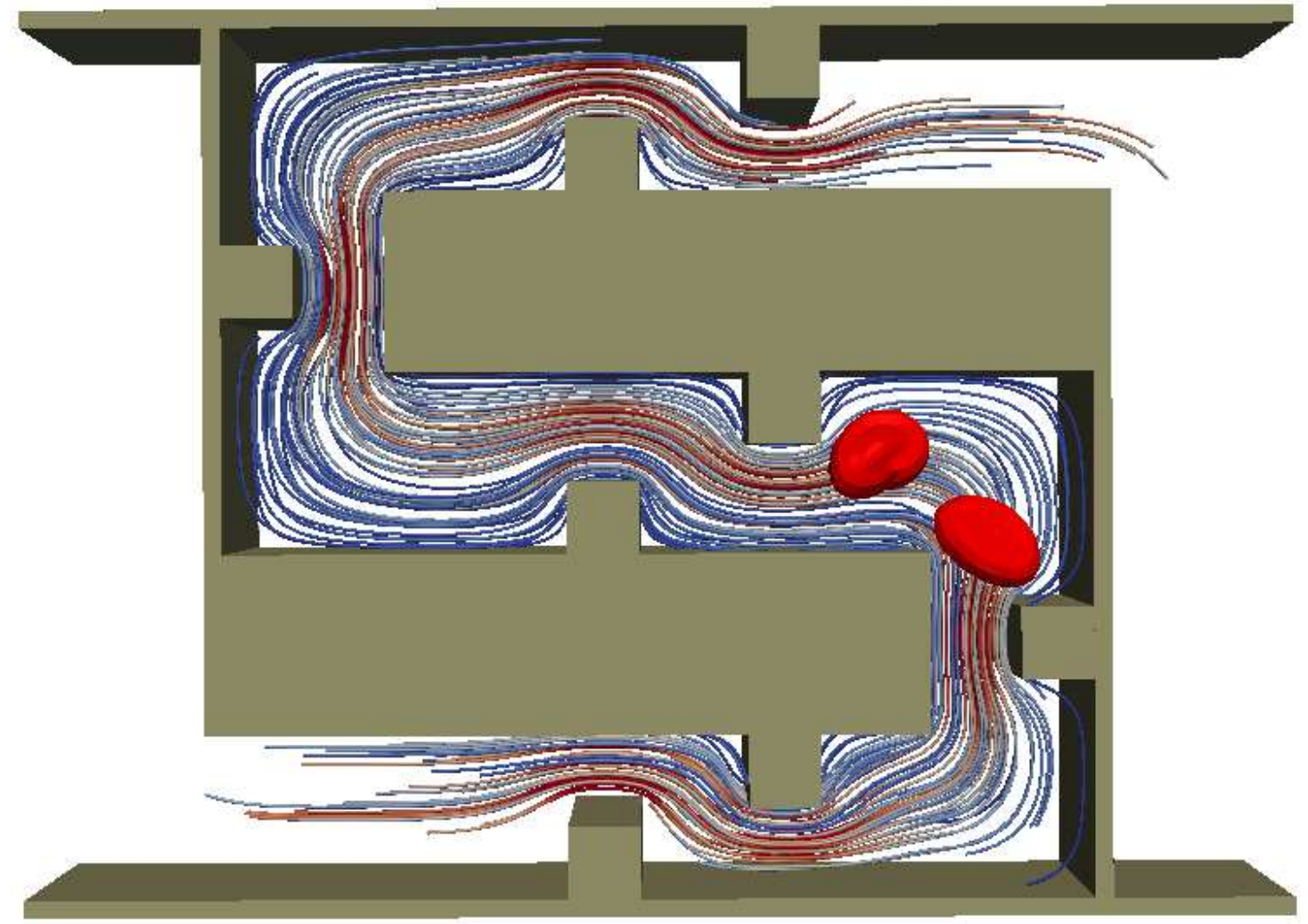

Figure 3: Two red blood cells passing an S-shaped microfluidic channel with obstacles. Fluidic streamlines show the increased velocity at narrow gaps (red). Simulation data produced by ESPResSo is visualized with ParaView [15].

\section{Conclusion}

Some publications already have appeared that use the OIF framework for simulations $[4,19,21]$. Currently, the OIF framework undergoes further developments. We are working on implementation of affinity mechanisms, so that in the future it will be possible to model the adhesion of cells to 
surfaces. We also plan to make the visualization of OIF data more convenient by implementing automatic generation of VTK files that can be easily viewed, e.g. in ParaView.

There have been collaborations started with biologists on simulating biological processes. Our aim is to make the OIF framework easy to use and well documented so that a scientist with basic programming skills will be able to master the Tcl code necessary to set up a simulation.

\section{References}

[1] R. Maniyeri, Y. K. Suh, S. Kang, M. J. Kim, Numerical study on the propulsion of a bacterial flagellum in a viscous fluid using an immersed boundary method, Computers \& Fluids 62 (2012) 13-24. doi:10.1016/j.compfluid.2012.03.012.

[2] A. Gilmanov, F. Sotiropoulos, A hybrid cartesian/immersed boundary method for simulating flows with 3D, geometrically complex, moving bodies, Journal of Computational Physics 207 (2) (2005) 457-492. doi:10.1016/j.jcp.2005.01.020.

[3] J. Chen, J. Li, Y. Sun, Microfluidic approaches for cancer cell detection, characterization, and separation, Lab on a Chip 12 (10) (2012) 1753-1767. doi:10.1039/C2LC21273K.

[4] M. Gusenbauer, H. Özelt, J. Fischbacher, F. Reichel, L. Exl, S. Bance, N. Kataeva, C. Binder, H. Brckl, T. Schrefl, Simulation of magnetic active polymers for versatile microfluidic devices, EPJ Web of Conferences 40 (2013) 02001. doi:10.1051/epjconf/20134002001.

[5] M. Gusenbauer, A. Kovacs, F. Reichel, L. Exl, S. Bance, H. Özelt, T. Schrefl, Self-organizing magnetic beads for biomedical applications, Journal of Magnetism and Magnetic Materials 324 (6) (2012) 977-982. doi:10.1016/j.jmmm.2011.09.034.

[6] Z. Guo, T. S. Zhao, Lattice boltzmann model for incompressible flows through porous media, Physical Review E 66 (3) (2002) 036304. doi:10.1103/PhysRevE.66.036304.

[7] M. E. Kutay, A. H. Aydilek, E. Masad, Laboratory validation of lattice boltzmann method for modeling pore-scale flow in granular materials, Computers and Geotechnics 33 (8) (2006) 381-395. doi:10.1016/j.compgeo.2006.08.002.

[8] S. Geller, M. Krafczyk, J. Tlke, S. Turek, J. Hron, Benchmark computations based on lattice-boltzmann, finite element and finite volume methods for laminar flows, Computers \& Fluids 35 (8-9) (2006) 888-897. doi:10.1016/j.compfluid.2005.08.009.

[9] D. Kandhai, A. Koponen, A. Hoekstra, M. Kataja, J. Timonen, P. Sloot, Lattice-boltzmann hydrodynamics on parallel systems, Computer Physics Communications 111 (1-3) (1998) 14-26. doi:10.1016/S0010-4655(98)000253.

[10] N. Satofuka, T. Nishioka, Parallelization of lattice boltzmann method for incompressible flow computations, Computational Mechanics 23 (2) (1999) 164-171. doi:10.1007/s004660050397.

[11] S. Williams, J. Carter, L. Oliker, J. Shalf, K. Yelick, Optimization of a lattice boltzmann computation on state-of-the-art multicore platforms, Journal of Parallel and Distributed Computing 69 (9) (2009) 762-777. doi:10.1016/j.jpdc.2009.04.002.

[12] C. S. Peskin, Flow patterns around heart valves: A numerical method, Journal of Computational Physics 10 (2) (1972) 252-271. doi:10.1016/0021-9991(72)90065-4.

[13] P. Ahlrichs, B. Dunweg, Lattice-Boltzmann simulation of polymer-solvent systems, Int. J. Modern Phys. C 8 (1998) 1429-1438.

[14] B. Dunweg, A. Ladd, Lattice boltzmann simulations of soft matter systems, Advances in Polymer Science 221. doi:http://dx.doi.org/10.1007/978-3-540-87706-6_2.

[15] ParaView - open source scientific visualization, http://www.paraview.org/, last visited on 21/06/2013.

[16] ESPResSo documentation, http://espressomd.org/wordpress/documentation/, last visited on 20/08/2013.

[17] H. Limbach, A. Arnold, B. Mann, C. Holm, ESPResSo- an extensible simulation package for research on soft matter systems, Computer Physics Communications 174 (2006) 704-727.

[18] A. Arnold, O. Lenz, S. Kesselheim, R. Weeber, F. Fahrenberger, D. Roehm, P. Košovan, C. Holm, ESPResSo 3.1 - molecular dynamics software for coarse-grained models, in: M. Griebel, M. Schweitzer (Eds.), Meshfree Methods for Partial Differential Equations VI, Lecture Notes in Computational Science and Engineering, Vol. 89, 2013, pp. 1-23.

[19] I. Cimrák, M. Gusenbauer, T. Schrefl, Modelling and simulation of processes in microfluidic devices for biomedical applications, Comput. Math. Appl. 64 (3) (2012) 278-288. doi:10.1016/j.camwa.2012.01.062. 
[20] M. Dao, C. Lim, S. Suresh, Mechanics of the human red blood cell deformed by optical tweezers, J. Mech. Phys. Solids 51 (2003) 2259-2280.

[21] I. Cimrák, I. Jančigová, K. Bachratá, H. Bachratý, On elasticity of spring network models used in blood flow simulations in ESPResSo, in: M. Bisschoff, E. Oñate, D. Owen, E. Ramm, P. Wriggers (Eds.), III International Conference on Particle-based Methods - Fundamentals and Applications PARTICLES 2013, 2013, pp. $133-144$.

[22] D. A. Fedosov, B. Caswell, G. E. Karniadakis, A multiscale red blood cell model with accurate mechanics, rheology, and dynamics, Biophysical Journal 98 (10) (2010) 2215-2225. doi:http://dx.doi.org/10.1016/j.bpj.2010.02.002.

URL http://www.sciencedirect.com/science/article/pii/S0006349510002286

[23] D. A. Fedosov, B. Caswell, G. E. Karniadakis, Systematic coarse-graining of spectrin-level red blood cell models, Computer Methods in Applied Mechanics and Engineering 199 (29-32) (2010) 1937-1948. doi:http://dx.doi.org/10.1016/j.cma.2010.02.001. URL http://www.sciencedirect.com/science/article/pii/S0045782510000599

[24] T. Odenthal, B. Smeets, P. Van Liedekerke, E. Tijskens, H. Van Oosterwyck, H. Ramon, Analysis of initial cell spreading using mechanistic contact formulations for a deformable cell model, PLoS Computational BiologyAccepted for publication.

[25] M. Dupin, I. Halliday, C. Care, L. Alboul, Modeling the flow of dense suspensions of deformable particles in three dimensions, Phys Rev E Stat Nonlin Soft Matter Phys. 75 (2007) 066707. 